\title{
Latent Class Trajectory Modeling of 2-Component Disease Activity Score in 28 Joints Identifies Multiple Rheumatoid Arthritis Phenotypes of Response to Biologic Disease-Modifying Antirheumatic Drugs
}

Arianna Dagliati, ${ }^{1}$ (DD Darren Plant, ${ }^{2}$ Nisha Nair, ${ }^{3}$ Meghna Jani, ${ }^{4}$ (DD Beatrice Amico, ${ }^{5}$ Niels Peek, ${ }^{6}$ Ann W. Morgan, ${ }^{7}$ John Isaacs, ${ }^{8}$ Anthony G. Wilson, ${ }^{9}$ iD Kimme L. Hyrich, ${ }^{10}$ Nophar Geifman, ${ }^{1}$ and Anne Barton, ${ }^{2}$ for the BRAGGSS Study Group

Objective. To determine whether using a reweighted disease activity score that better reflects joint synovitis, i.e., the 2-component Disease Activity Score in 28 joints (DAS28) (based on swollen joint count and C-reactive protein level), produces more clinically relevant treatment outcome trajectories compared to the standard 4-component DAS28.

Methods. Latent class mixed modeling of response to biologic treatment was applied to 2,991 rheumatoid arthritis (RA) patients in whom treatment with a biologic disease-modifying antirheumatic drug was being initiated within the Biologics in Rheumatoid Arthritis Genetics and Genomics Study Syndicate cohort, using both 4-component and 2-component DAS28 scores as outcome measures. Patient groups with similar trajectories were compared in terms of pretreatment baseline characteristics (including disability and comorbidities) and follow-up characteristics (including antidrug antibody events, adherence to treatments, and blood drug levels). We compared the trajectories obtained using the 4- and 2-component scores to determine which characteristics were better captured by each.

Results. Using the 4-component DAS28, we identified 3 trajectory groups, which is consistent with previous findings. We showed that the 4-component DAS28 captures information relating to depression. Using the 2-component DAS28, 7 trajectory groups were identified; among them, distinct groups of nonresponders had a higher incidence of respiratory comorbidities and a higher proportion of antidrug antibody events. We also identified a group of patients for whom the 2-component DAS28 scores remained relatively low; this group included a high percentage of patients who were nonadherent to treatment. This highlights the utility of both the 4- and 2-component DAS28 for monitoring different components of disease activity.

Conclusion. Here we show that the 2-component modified DAS28 defines important biologic and clinical phenotypes associated with treatment outcome in RA and characterizes important underlying response mechanisms to biologic drugs.

Supported by the NIHR Manchester Biomedical Research Centre, Manchester Molecular Pathology Innovation Centre (grant MR/N00583X/1), NIHR Newcastle Biomedical Research Centre, and Versus Arthritis (grants 21754 and 22072). MATURA was jointly funded by the Medical Research Council and Versus Arthritis (grant MR/K015346/1). Drs. Isaacs and Barton are NIHR senior investigators.

'Arianna Dagliati, PhD, Nophar Geifman, PhD: Centre for Health Informatics and Manchester Molecular Pathology Innovation Centre, University of Manchester, Manchester, UK; ${ }^{2}$ Darren Plant, PhD, Anne Barton, PhD: Versus Arthritis Centre for Genetics and Genomics, University of Manchester, NIHR Manchester Biomedical Research Centre, Manchester University NHS Foundation Trust, and Manchester Academic Health Science Centre, Manchester, UK; ${ }^{3}$ Nisha Nair, PhD: Versus Arthritis Centre for Genetics and Genomics, University of Manchester, Manchester, UK; ${ }^{4}$ Meghna Jani, PhD: Versus Arthritis Centre for Epidemiology, University of Manchester, Manchester, UK; ${ }^{5}$ Beatrice Amico, MS: University of Verona, Verona, Italy; ${ }^{6}$ Niels Peek, PhD: Centre for Health Informatics, University of Manchester, NIHR Manchester Biomedical Research Centre, Manchester University NHS Foundation Trust, and Manchester Academic Health Science Centre, Manchester, UK; ${ }^{7}$ Ann W. Morgan, PhD: University of Leeds School of Medicine, NIHR Leeds Biomedical Research Centre, and Leeds Teaching Hospitals NHS Trust, Leeds, UK; ${ }^{8}$ John Isaacs, PhD: Translational and Clinical Research Institute, Newcastle University, and Newcastle upon Tyne Hospitals NHS Foundation Trust, Newcastle upon Tyne, UK; ${ }^{9}$ Anthony G. Wilson, PhD: Centre for Arthritis Research, Conway Institute, University College Dublin, Dublin, Ireland; ${ }^{10}$ Kimme L. Hyrich, PhD: NIHR Manchester Biomedical Research Centre, Manchester University NHS Foundation Trust, Manchester Academic Health Science Centre, and Versus Arthritis Centre for Epidemiology, University of Manchester, Manchester, UK.

Drs. Dagliati and Plant contributed equally to this work. Drs. Geifman and Barton contributed equally to this work.

No potential conflicts of interest relevant to this article were reported.

Address correspondence to Anne Barton, PhD, The University of Manchester, Oxford Road, Manchester M13 9PT, UK. Email: anne.barton@ manchester.ac.uk.

Submitted for publication January 29, 2020; accepted in revised form May 21, 2020. 


\section{INTRODUCTION}

Complexity and variability of patient trajectories in response to treatment pose significant challenges in many medical fields (1-4). A current focus of medical research is facilitating a shift away from a "one-size-fits-all" approach to precision medicine approaches aimed at identifying narrower patient subgroups that would benefit from tailored interventions. However, the high variability in response to therapeutic agents over time makes prediction of response challenging (5-7).

Among subgroup discovery approaches using longitudinal data, latent class mixed modeling (LCMM) is gaining increasing popularity for identifying homogeneous subgroups (8-12). LCMM has been applied to study disease progression and response to treatments in psychiatry, nephropathy, neurology (13-15), and early arthritis (16). In rheumatoid arthritis (RA), LCMM has been used to identify trajectories of disability progression following diagnosis of RA (17). Outcome measures in previous studies have included the Disease Activity Score in 28 joints (DAS28) (18-21) for treat-to-target strategies (19), response to biologic agents (20), and prediction of remission in patients treated with disease modifying antirheumatic drugs (DMARDs) (21). All of these studies used the 4-component DAS28 and identified 3 broad trajectories.

It has previously been demonstrated that the 4-component DAS28 correlates poorly with synovitis (22). Given that the drugs used to treat RA aim to reduce synovial inflammation and prevent joint damage, it is unclear how useful the 4-component DAS28 trajectories will be as outcome measures for precision medicine studies, which aim to identify biomarkers that are predictive of therapeutic response. To address this, a revised composite disease activity score, including only the swollen joint count (SJC) and C-reactive protein (CRP) levels (2-component DAS28), has been developed and shown to better correlate with ultrasounddetected synovial inflammation compared to the original 4-component activity score (22).

We undertook this study to apply the LCMM approach in a prospective, longitudinal cohort of patients beginning biologic DMARD treatment. We used the original 4-component DAS28 and 2-component DAS28 in order to assess whether the identified trajectory groups have clinical relevance, by exploring their association with comorbidities, treatment adherence, and drug immunogenicity, all of which have previously been reported to be associated with biologic DMARD treatment response $(23,24)$.

\section{PATIENTS AND METHODS}

Study patients. We used longitudinal data (November 2008-January 2018) from patients recruited into the Biologics in Rheumatoid Arthritis Genetics and Genomics Study Syndicate (BRAGGSS), an observational multicenter study in the UK established to study predictors of treatment response to biologic DMARDs (25). Consenting participants were eligible if they had a consultant diagnosis of RA, were $>18$ years old, and were about to initiate treatment with biologic DMARDs, namely tumor necrosis factor (TNF), B cell inhibitor (anti-CD20), interleukin-6 receptor (IL-6R), or T cell inhibitor (CTLA-4). Information regarding the combination with conventional DMARDs, e.g., methotrexate (MTX), was recorded. During the observational study, biologic DMARD therapy was suspended, changed, or restarted in a minority of patients. These patients were included in the analyses, and information about previous treatment with biologic DMARDs was recorded. Following the pretreatment baseline sample and data collection, patients were observed for 12 months, with follow-up visits at 3,6 , and 12 months. Participating patients provided written informed consent, and the BRAGGSS study was approved by the local ethics committee (COREC 04/Q1403/37).

The primary outcome measure to model response trajectories was the disease activity score based on the 2-component DAS28, computed as

$$
\text { sqrt }(\mathrm{SJC})+\left(0.6^{*} \ln [\mathrm{CRP}+1]\right) \text {. }
$$

Four-component DAS28 score and response criteria were calculated according to standard methodology (26). Variables recorded at baseline included age, sex, time since diagnosis, body mass index (BMI), smoking status (if the patient had ever smoked), functional severity measured by the Health Assessment Questionnaire (HAQ) (27), and consultant-reported comorbidities. DAS28 components (tender joint count [TJC], SJC, patient global assessment of well-being measured on a 100-mm visual analog scale [VAS], serum CRP level) were measured at baseline (i.e., before commencing any biologic DMARD treatment) and at each follow-up visit. For patients treated with TNF inhibitors, antidrug antibodies and serum drug levels were measured in serum samples collected at follow-up visits.

We included patients with $\geq 1$ score from the 2-component DAS28 after baseline, including all measurements until the last registered follow-up. Unlike previous studies $(28,29)$, we did not exclude patients on the basis of the number of scores, in order to avoid possible selection biases, and we also included patients who had received biologic treatment in the past. Data on patients who switched biologic DMARDs were right-censored when the change occurred. Missing data arose from noncompletion of responses to questions on demographic characteristics and missing $\mathrm{HAQ}$ scores. Due to the nature of the variables, we assumed they were missing at random. As in previous studies $(30,31)$, we compared different imputation strategies (see Supplementary Methods, on the Arthritis \& Rheumatology website at http://onlinelibrary.wiley.com/doi/10.1002/art.41379/ abstract) and implemented the method with the best performance. Data were imputed with a random forest approach, with 100 trees and a maximum of 100 iterations.

LCMM. We applied LCMM to identify patient subgroups with distinct responses to biologic DMARDs over time. Previous stratification studies in RA using LCMM focused on the 4-component DAS28 as the measure of disease activity and response to 
biologic treatment (18-21); those analyses identified 3 groups of responders: rapid, gradual, and inadequate. In the present study, we specified 2 linear mixed-effects models: the 4-component DAS28 used in previous studies and the 2-component DAS28 used here as the dependent variable.

Mixed effects were used to account for the likely correlation of repeated measurements and included a random intercept for each individual. We fitted the model through the "Icmm" function of the R package Icmm (32). We followed the framework described by Lennon et al (33) that includes scoping model definition, refinement of the number of classes and model structure, model adequacy assessment and clinical plausibility, graphic presentations, and sensitivity analysis (Supplementary Methods, http://online library.wiley.com/doi/10.1002/art.41379/abstract).

Models were adjusted for age, time since diagnosis, sex, BMI, HAQ score, biologic DMARD therapy, concomitant treatment with MTX, and previous treatment with biologic drugs. We developed scoping models for 1-10 classes to determine the optimal number of classes based on the Bayesian information criterion (BIC) and further refined the models comparing linear, quadratic, and cubic specifications of time (Supplementary Methods, http://online library.wiley.com/doi/10.1002/art.41379/abstract).

To evaluate whether results were driven by differential relative effectiveness of therapies, in sensitivity analyses we repeated the analysis in patients treated with TNF inhibitors only and in those treated with any biologic DMARD in combination with MTX, comparing the discovered latent classes in terms of baseline characteristics and response variables.

Statistical analysis and comparison of latent classes. Discovered latent classes were described and compared in terms of clinical characteristics, comorbidities, adherence to treatment, antidrug antibody positivity, and non-trough blood drug levels.

Treatment response variation in individual patients. To describe variation in treatment responses in each patient over time, we computed slopes as the difference between consecutive measurements divided by the time between the measurements (Supplementary Methods, http://onlinelibrary.wiley.com/ doi/10.1002/art.41379/abstract). Higher slope values indicate deterioration of a patient's condition, while negative values indicate improvements. Time to nonresponse was defined as a switch from a negative to a positive value of the slope (i.e., when the slopes start to increase). Intercept values and slopes were compared between classes using analysis of variance (ANOVA). Time to nonresponse was compared among nonresponders using Kaplan-Meier curves.

Demographic and clinical characteristics. Clinical characteristics were compared among latent classes. Categorical characteristics (sex, pretreatment smoking status, MTX cotherapy, and switch of biologic DMARDs) were compared using chi-square tests followed by Bonferroni-Holm correction, while continuous variables (age, disease duration, HAQ score, BMI, and DAS28 score) were compared using ANOVA with Tukey's post hoc test.

Association of latent classes with comorbidities and therapy (TNF inhibitor, B cell inhibitor, IL-6R inhibitor, and T cell inhibitor) was explored using logistic regression, where the latent class assignment was the regression predictor, and least squares means was used for multiple comparisons with Tukey's adjustment (from the $\mathrm{R}$ Ismeans package [34]). The effect of respiratory comorbidities on latent classes was assessed by stratified analyses in smokers (current or past) and nonsmokers (never).

Treatment adherence, drug levels, and antidrug antibody positivity. We examined the associations of latent class subgroup with treatment adherence, drug levels, and antidrug antibody positivity. Drug level and antidrug antibody values have previously been shown to be associated with response to biologic DMARDs (24). Self-reported adherence was recorded at 3 and 6 months following the start of therapy (35); adherent patients were defined as those who took the dose on the day agreed upon by their health care team or $\leq 1$ day before or after, while nonadherent patients were defined as those who self-reported either missing the dose completely or taking it $>1$ day before or after the agreed day.

The correlation between drug levels and latent classes was investigated in terms of patient-level relative change at the next follow-up visit. We created matched samples with propensity scoring, including BMI (36), age, and sex as potential confounders without interactions and drug level changes (increasing or decreasing levels) as a binary outcome measure. Chi-square tests were then applied to assess differences in drug level changes among latent classes.

To evaluate antidrug antibody development, we used a threshold of 12 arbitrary/ml as a positive event (24). Heterogeneities of latent classes across the total cohort and the subset with antidrug antibody information were determined by Cochran's $Q$ statistic. Antidrug antibody development events were compared in latent classes using Kaplan-Meier curves and Cox proportional hazards regression models, with adjustment for age, sex, BMl, and concomitant treatment with MTX.

Analyses were computed using $\mathrm{R}$, version 3.2.3. Data are presented as the main effect with 95\% confidence intervals (95\% Cls), and the $5 \%$ significance level was used for main inferences.

\section{RESULTS}

Patient characteristics. Our data set included 2,991 patients with 7,567 DAS28 measurements after baseline, excluding the baseline measure. In the 12-month observation period from baseline, patients had 1-3 follow-up visits: 1,647 patients (55.07\%) had 1 follow-up visit, 661 (22.10\%) had 2 follow-up visits, and 683 (22.84\%) had 3 follow-up visits. The first follow-up visit took place a mean \pm SD of $124 \pm 51$ days after baseline, the second $217 \pm 51$ days after baseline, and the third $365 \pm 50$ days after baseline. Table 1 presents baseline characteristics, including percentages of missing data. 
Table 1. Baseline characteristics of the cohort $(n=2,991)^{\star}$

\begin{tabular}{lc}
\hline Age, years & $62.18 \pm 12.46$ \\
Time from diagnosis, years & $10.46 \pm 9.75$ \\
Height, cm & $164.49 \pm 11.83$ \\
Weight, kg & $79.24 \pm 19.81$ \\
HAQ score & $1.72 \pm 0.60$ \\
Male sex, no. (\%) & $710(23.74)$ \\
Ever-smoker, no. (\%) & $1,868(73.00)$ \\
2-component DAS28 score & $4.25 \pm 1.19$ \\
4-component DAS28 score & $5.65 \pm 0.96$ \\
TJC & $14.56 \pm 7.26$ \\
SJC & $8.35 \pm 5.35$ \\
VAS score & $72.01 \pm 19.49$ \\
CRP, mg/liter & $20.49 \pm 29.10$ \\
Treatment regimen & \\
Biologic and nonbiologic DMARDs, no. (\%) & $559(18.69)$ \\
Biologic and nonbiologic DMARDs (including & $1,878(62.79)$ \\
MTX), no. (\%) & \\
Only biologic DMARDs, no. (\%) & $554(18.52)$ \\
Previous treatment with biologics, no. (\%) & $673(22.5)$ \\
Type of biologic & \\
TNF inhibitor, no. (\%) & $2,151(71.92)$ \\
Adalimumab, no. & 664 \\
Certolizumab, no. & 373 \\
Etanercept, no. & 904 \\
Other, no. & 210 \\
B cell inhibitor, no. (\%) & $436(14.58)$ \\
IL-6R inhibitor, no. (\%) & $278(9.29)$ \\
T cell inhibitor, no. (\%) & $123(4.11)$ \\
Other, no. (\%) & $3(0.10)$ \\
\hline
\end{tabular}

* Except where indicated otherwise, values are the mean \pm SD. Data were missing for the following number of patients in specified categories: age $(n=12)$, time from diagnosis $(n=157)$, height $(n=589)$, weight $(n=197)$, Health Assessment Questionnaire (HAQ) score $(n=475)$, and smoking status $(n=432)$. DAS28 = Disease Activity Score in 28 joints; TJC = tender joint count; $\mathrm{SJC}=$ swollen joint count; VAS = visual analog scale; CRP = C-reactive protein; DMARD = disease-modifying antirheumatic drug; $\mathrm{MTX}=$ methotrexate; $\mathrm{TNF}=$ tumor necrosis factor; IL-6R = interleukin-6 receptor.

Four-component DAS28 trajectories and comparisons to previous studies. We reproduced a 3-trajectory model using the 4-component DAS28, with 3 classes producing the lowest $\mathrm{BIC}$ and highest assignment posterior probability compared to models with a range of 1-10 classes (Supplementary Figure 18 and Supplementary Table 13, http://onlinelibrary.wiley.com/ doi/10.1002/art.41379/abstract).

Although inclusion criteria of previous studies were different $(19,21)$ and the mean 4-component DAS28 score at baseline was higher (20), response patterns in the present study were qualitatively comparable. We identified the following groups: a rapid responder group $(n=2,004$ [67.00\%]) with quick improvement in the first observation period followed by stabilization or slight increases in disease activity, a gradual responder group $(n=919$ [30.73\%]) with slower but consistent decrease in disease activity, and a poor responder group $(n=68$ [2.27\%]) (Supplementary Figure 19 and Supplementary Table 14, http://onlinelibrary.wiley. com/doi/10.1002/art.41379/abstract).

These 4-component DAS28 classes were compared to response patterns and classifications based on European League
Against Rheumatism (EULAR) response criteria for RA (37) at different time points. As expected, grouping by LCMM and EULAR criteria revealed proportional differences, with the majority of patients in the $3 \mathrm{LCMM}$ classes grouped as either nonresponders or intermediate responders according to the EULAR criteria. The comparison of EULAR response classes between the latent classes confirmed the following findings: EULAR nonresponse was reached by the majority of LCMM poor responders by the third follow-up; LCMM gradual responders had a high percentage of EULAR moderate responders, with $>50 \%$ of the population being classified as moderate responders by the third follow-up; the majority of LCMM rapid responders were classified as EULAR good responders by the third follow-up (Supplementary Methods, Supplementary Table 15, and Supplementary Figure 20, http:// onlinelibrary.wiley.com/doi/10.1002/art.41379/abstract).

Comparisons between the latent trajectory groups defined using the 4-component DAS28 identified differences related to depression, extracted from reported comorbidities, with borderline statistical evidence for higher rates in gradual responders compared to rapid responders (398 of 2,004 rapid responders [19.9\%] and 214 of 919 gradual responders [23.3\%]; odds ratio $[\mathrm{OR}] 1.22$, risk ratio 1.05, $P=0.07$ ) (Supplementary Figure 21 and Supplementary Table 16, http://onlinelibrary.wiley.com/doi/10. 1002/art.41379/abstract).

We found no significant correlation between drug level variation and response trajectories using the 4-component DAS28 $(P=0.40)$. Data on antidrug antibodies were available for 475 patients receiving adalimumab or certolizumab, whose disease activity classes distributed in a similar manner to that of the whole cohort (Supplementary Table 12, http://onlinelibrary.wiley.com/ doi/10.1002/art.41379/abstract). Antidrug antibody positivity was higher in gradual responders compared to rapid responders ( $\beta=1.79$ [95\% Cl 1.30-2.46], $P=0.001$ ) (Supplementary Figures 23 and 24, http://onlinelibrary.wiley.com/doi/10.1002/art.41379/ abstract).

Seven response trajectory groups identified by 2-component DAS 28 latent class analysis. Two-component DAS28-based LCMM analysis identified 7 trajectories, which were arbitrarily labeled according to the score at pretreatment and change in score over time (Supplementary Methods, http://online library.wiley.com/doi/10.1002/art.41379/abstract). These trajectory groups included the following: 2 groups of good responders, 2 groups of gradual responders, 2 groups of secondary nonresponders, and 1 group of low disease activity (Figure 1). Individual DAS28 component trajectories in the discovered latent classes are shown in Supplementary Figure 12 (http://onlinelibrary.wiley. com/doi/10.1002/art.41379/abstract).

Good responders demonstrated fast improvement in disease activity in the first 3 months, followed by maintenance of good response (Figure 1). The first group of good responders ( $n=395$ [13.2\%]) and the second group $(n=1,840$ [61.5\%]) differed 


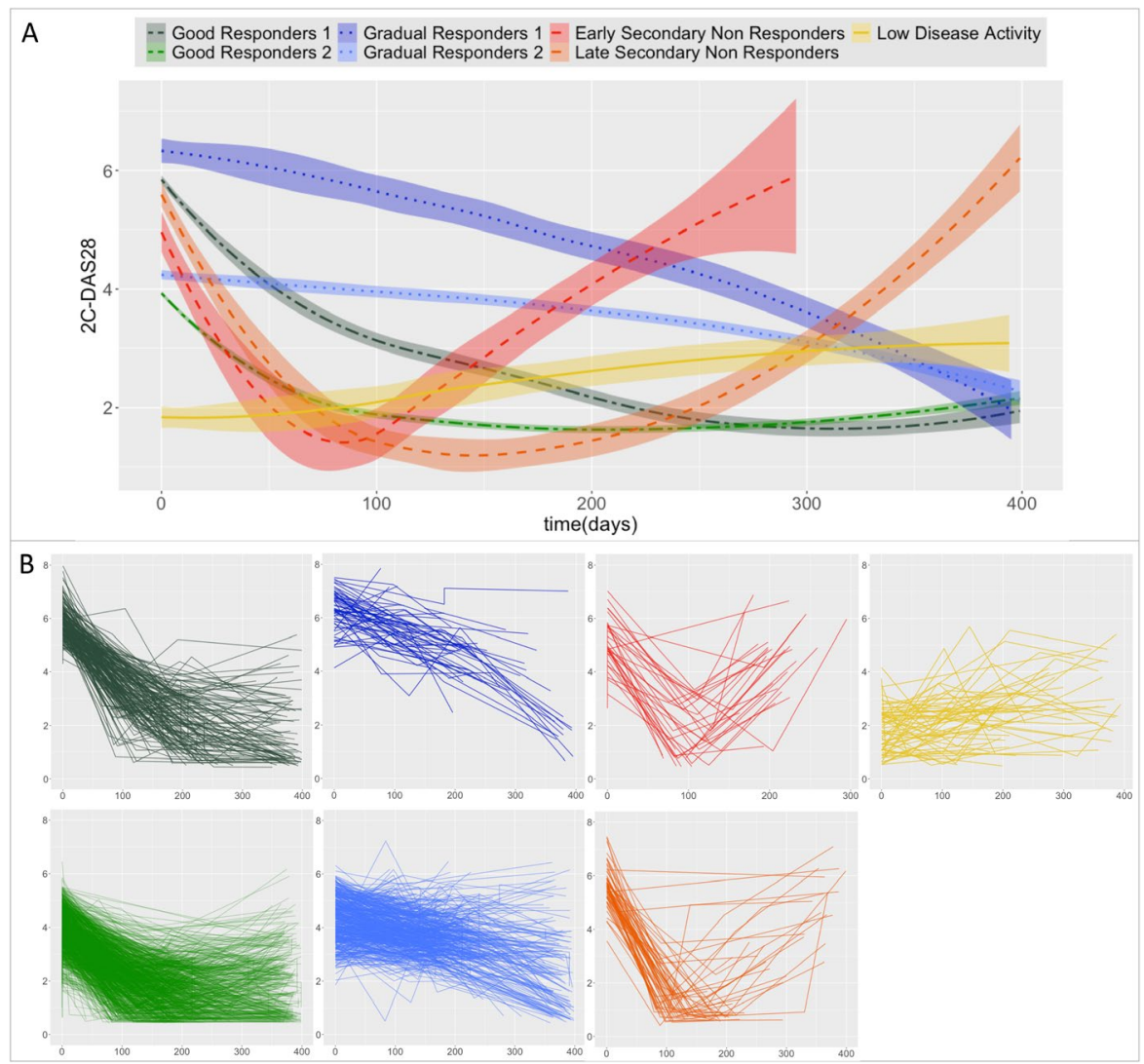

Figure 1. Latent class mixed modeling trajectories. A, Two-component Disease Activity Score in 28 joints (2C-DAS28)-based trajectories, with 95\% confidence intervals, in patients in each discovered latent class, determined using the loess method. B, Individual patient trajectories in each identified latent class.

significantly in baseline score for the 2-component DAS28, with the first group showing higher values (mean \pm SD $5.85 \pm 0.57$ ) compared to the second group (mean \pm SD $3.94 \pm 0.79)\left(P<2.2 \times 10^{-16}\right)$.
Gradual responders demonstrated a continuous but gradual reduction in disease activity (Figure 1). These 2 groups also had significantly different baseline scores for the 2-component DAS28,

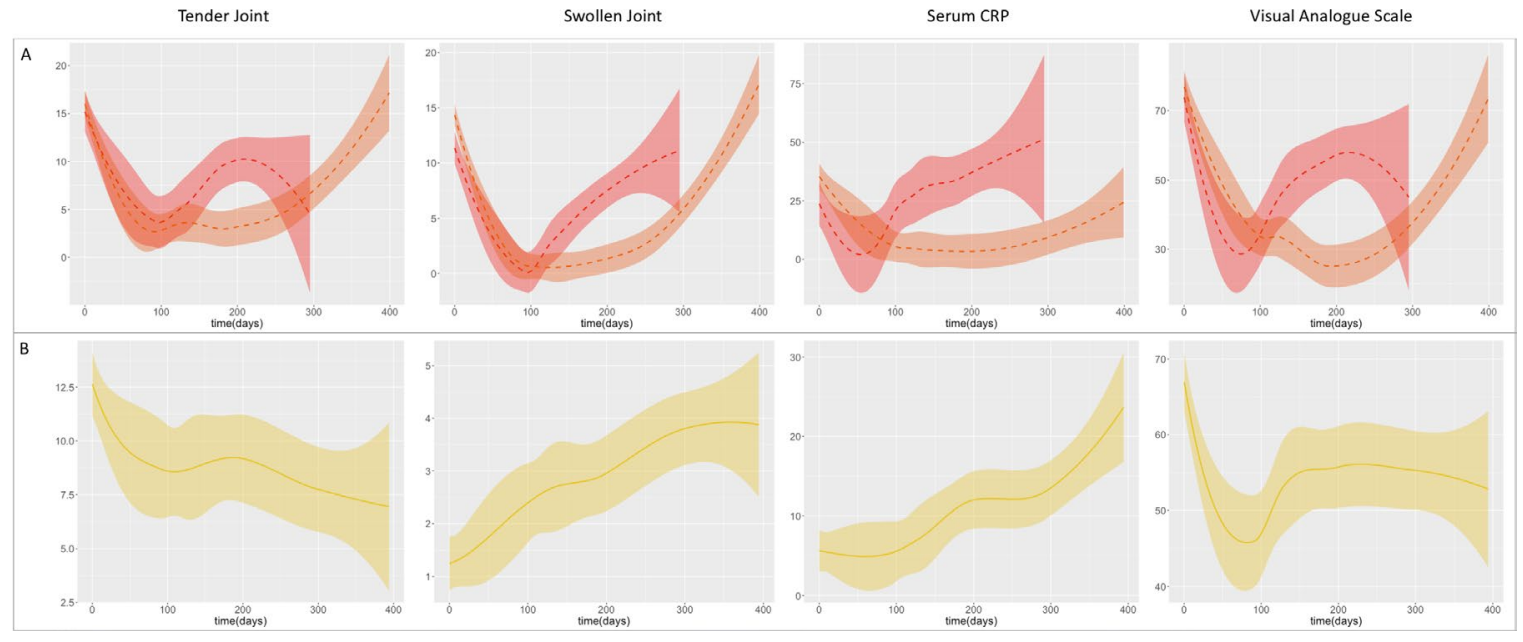

Figure 2. Four-component Disease Activity Score in 28 joints (DAS28) in 3 subgroups. Four-component DAS28 scores in the secondary nonresponders groups $(\mathbf{A})$ and the low activity disease group (B) with 95\% confidence intervals are shown. Score curves were determined using the loess method. In early secondary nonresponders (red), both swollen joint count (SJC) and C-reactive protein (CRP) levels followed the same pattern detected with the 2-component DAS28. In late secondary nonresponders (orange), the nonresponse pattern was mainly driven by the SJC component, while the CRP pattern was mainly flat. The trajectories of the low activity disease group (yellow) showed high tender joint counts and visual analog scale scores, and the disease activity was mainly driven by these 2 components. 


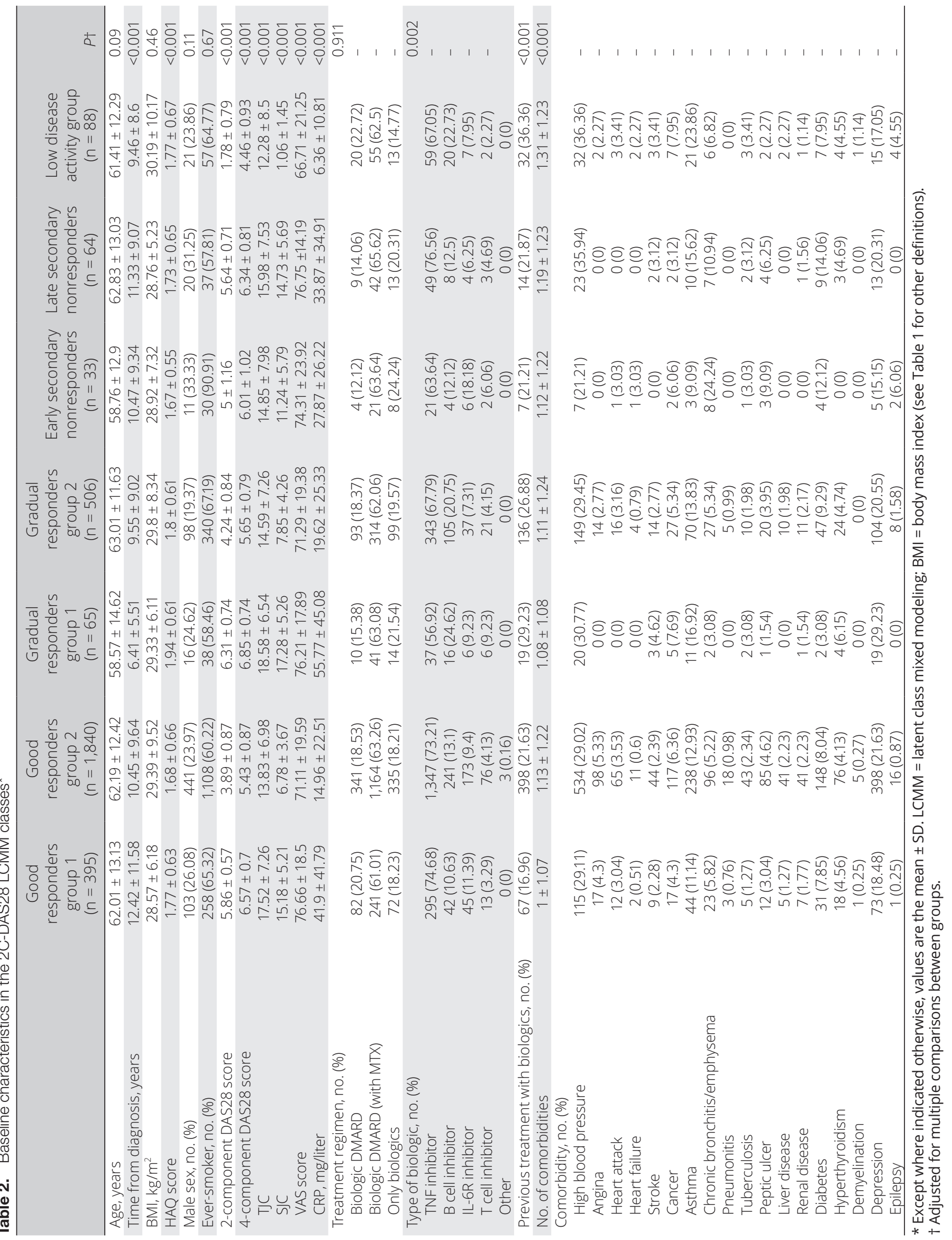


with the first group $(n=65$ [2.2\%]) having higher scores (mean \pm SD $6.33 \pm 0.79)$ than the second group $(n=506$ [16.9\%]; mean \pm SD $4.24 \pm 0.85)\left(P<2.2 \times 10^{-16}\right)$.

Secondary nonresponders showed a fast improvement in disease activity scores followed by rapid deterioration (Figure 1). This class encompassed 2 subgroups: early secondary nonresponders $(n=33[1.1 \%])$ and late secondary nonresponders ( $n=64$ [2.1\%]). Early nonresponders had significantly faster slopes of deterioration (difference 0.072, lower limit 0.001, upper limit 0.144; $P=0.044$ ) compared to late nonresponders.

Patients in the low disease activity group ( $n=88$ [2.9\%]) demonstrated the lowest DAS28 scores at baseline (mean \pm SD $2.24 \pm 1.09$ ), which increased modestly during follow-up (Figure 1). Initial levels of disease activity measured using the 4-component DAS28 were driven by TJC and VAS score, while SJC and CRP level showed a consistent increase in the follow-up period (Figure 2).

Latent classes were described in terms of slopes (Supplementary Figure 10 and Supplementary Table 6, http://onlinelibrary. wiley.com/doi/10.1002/art.41379/abstract). When we compared the 2 subgroups of secondary responders in terms of 2-component DAS28 score slopes and time to nonresponse, we found that early secondary nonresponders lost response significantly earlier (mean \pm SD $212 \pm 28$ days after the baseline visit) than late secondary nonresponders (mean \pm SD $265 \pm 73$ days) ( $P=0.005$ ) (Supplementary Figure 11, http://onlinelibrary.wiley. com/doi/10.1002/art.41379/abstract).

Patients in the low disease activity group showed modest improvements in TJC and VAS score during the observation period but demonstrated deterioration in SJC and CRP level (Figure 2). The majority of patients in the low disease activity group were classified as nonresponders (79\% by 6 months) using the 4-component DAS28-derived EULAR response criteria (Supplementary Tables 8 and 9, http://onlinelibrary.wiley.com/ doi/10.1002/art.41379/abstract). Importantly, the low disease activity group included the highest proportion of patients who had received other biologic DMARD treatments in the past (36.36\%).
Sensitivity analyses showed that in TNF inhibitor-treated patients ( $n=2,151$ ), the best model identified 6 classes, with an absence of the gradual responder class with low initial 2-component DAS28 score (Supplementary Figure 7, http://online library.wiley.com/doi/10.1002/art.41379/abstract). In patients treated concomitantly with TNF inhibitors and MTX ( $n=1,878)$, the best fitted model included 7 classes, with trajectories comparable to those in the whole-cohort model (Supplementary Figure 4, http://onlinelibrary.wiley.com/doi/10.1002/art.41379/abstract).

Two-component DAS28 response trajectories are associated with different baseline characteristics. LCMM analysis revealed significant differences among classes in time from diagnosis, HAQ scores, and number of comorbidities. Basic statistics and $P$ values are reported in Table 2. Further details can be found in the Baseline Characteristics section of the Supplementary Methods (http://onlinelibrary.wiley.com/doi/10.1002/art.41379/ abstract).

Figure 3 illustrates comorbidity prevalence in LCMM classes. Early secondary nonresponders had a higher prevalence of chronic bronchitis/emphysema compared to good responders (good responders group 1 OR 5.38, $P=0.005$; group 2 OR 5.85; $P=0.005$ ) and gradual responders (gradual responders group 2 OR 5.54, $P=0.003$ ). The stratified analysis of correlation between being an early secondary nonresponder and having chronic bronchitis/emphysema showed significant effects in ever-smokers (OR 2.49 [95\% Cl 1.14-5.42]) and never-smokers (OR 4.87 [95\% Cl 1.06-22.32]). Low disease activity patients had a higher prevalence of asthma compared to good responders (good responder group 1, OR $2.53 P=0.029$ ).

Two-component DAS28 response trajectories are associated with adherence, drug levels, and antidrug antibodies. Self-reported adherence measures, collected at follow-up visits, were available for 1,528 patients (51\%). Twocomponent DAS28 slopes decreased more quickly in adherent

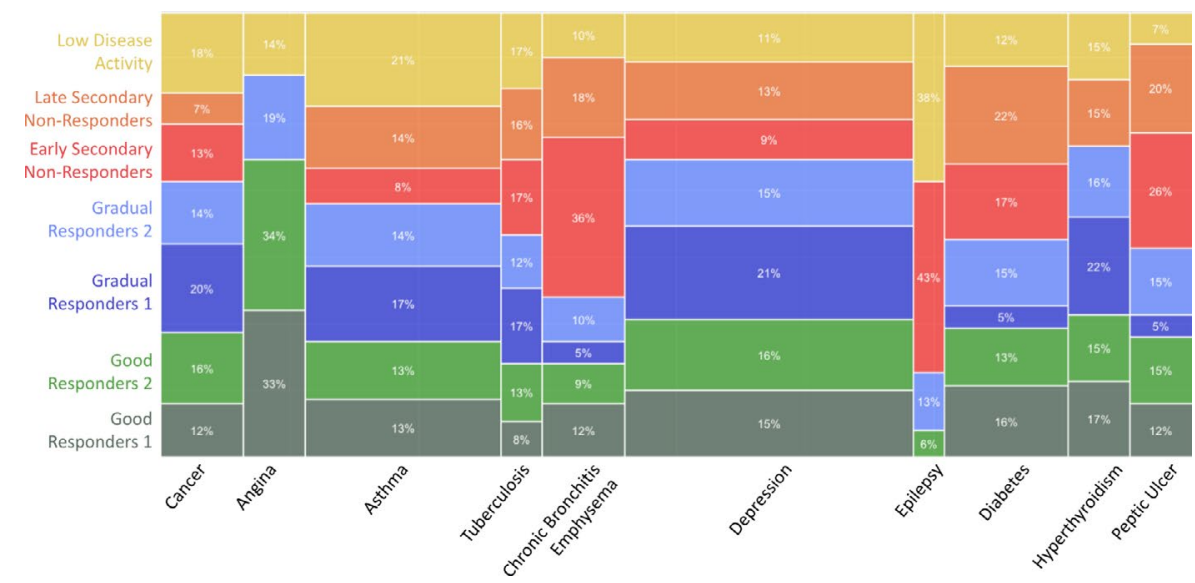

Figure 3. Comorbidity prevalence in the latent class mixed modeling subgroups. The width of the columns indicates the prevalence of each comorbidity across the whole population. 
patients (mean $\pm \mathrm{SD}-0.00128 \pm 0.0581$ ) than in nonadherent patients (mean $\pm \mathrm{SD}-0.0120 \pm 0.103 ; P=0.002$ ). Adherence patterns indicated that the low disease activity group, which were identified using only the 2-component DAS28, were the least adherent group (Supplementary Figure 16, http://onlinelibrary. wiley.com/doi/10.1002/art.41379/abstract).

Differences in adherence were confirmed by blood drug level changes (Supplementary Figure 17 and Supplementary Table 11, http://onlinelibrary.wiley.com/doi/10.1002/art.41379/abstract). Significant correlations between drug level changes at the second follow-up visit and 2-component DAS28 response trajectories were observed in the matched data set ( $n=524$ subjects) $(P=0.03)$.

The highest proportion of antidrug antibody-positive patients was observed in the early secondary nonresponders, but high antidrug antibody titers were also observed in gradual responders (Figure 4A). Cox regression models, which included the effect of MTX co-reatment, revealed different risks for antidrug antibodies $\left(\beta=6.06\right.$ [95\% Cl 2.57-14.27], $P=3.71 \times 10^{-5}$ for late secondary nonresponders compared to good responders group $1 ; \beta=1.79$ [95\% Cl 1.00-3.22], $P=0.018$ for gradual responders group 2 compared to good responders group 1) (Figure 4B).

\section{DISCUSSION}

In the present study, we determined that using the 2-component DAS28 score as an outcome measure revealed more subgroups of patients compared to the 4-component DAS28, and the trajectories identified were clinically meaningful. While the 4-component DAS28 remains an essential tool to

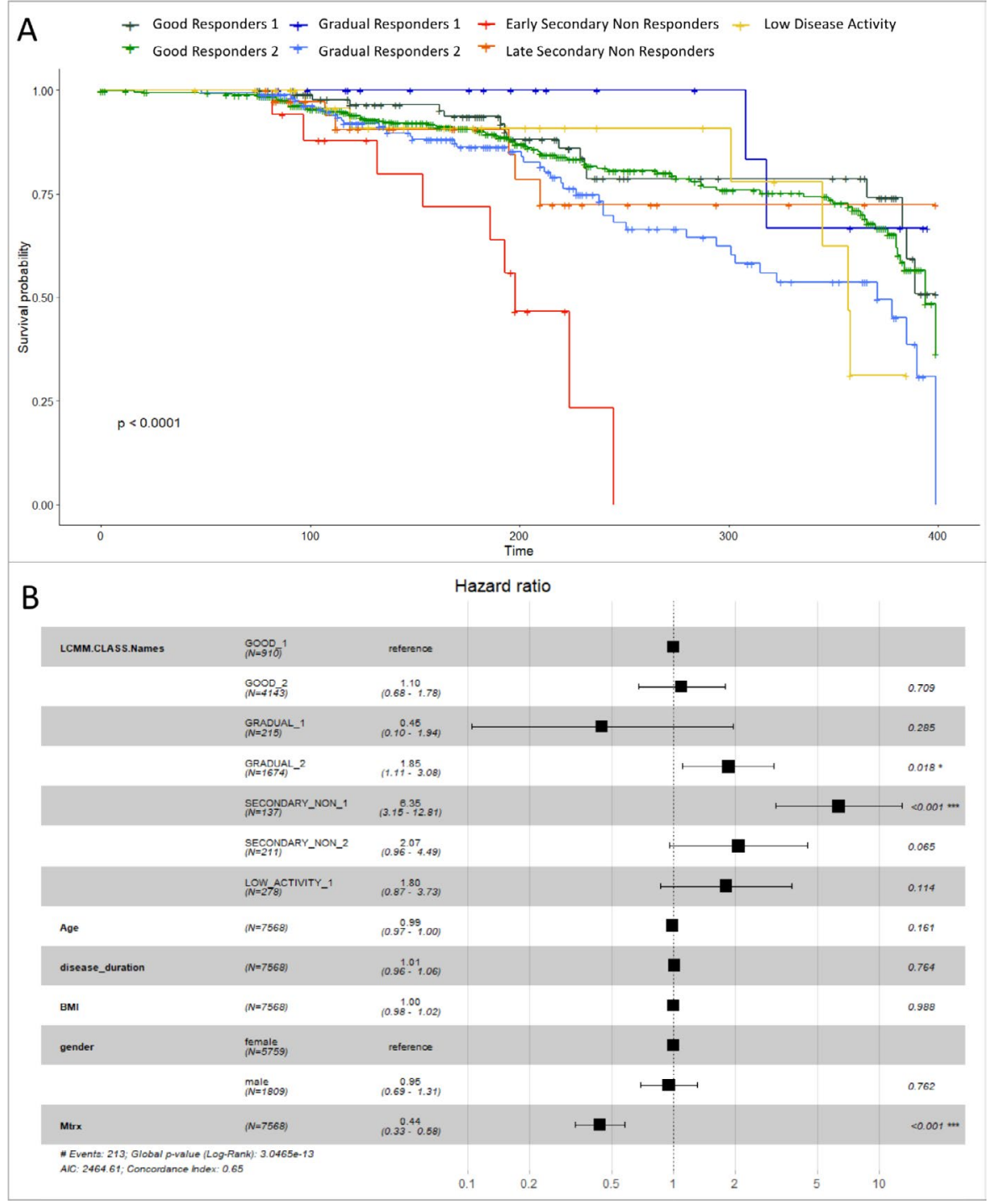

Figure 4. Antidrug antibody positivity. A, Kaplan-Meier curves of antidrug antibody positivity in latent class mixed modeling (LCMM) subgroups. Data from follow-up visits were censored at the last registered visit. B, Cox regression model for antidrug antibody positivity. Values in parentheses (depicted by bars) are the 95\% confidence intervals. BMI = body mass index; Mtrx = methotrexate; AIC = Akaike's information criterion. 
assess RA progression in clinical practice, the 3 trajectories identified, which can be also interpreted in terms of EULAR response criteria, may not provide sufficient granularity to help elucidate important biologic mechanisms involved in inflammation.

Strengths of this study include the fact that it is the first and largest study, based on real-world patients, to explore patientcentered factors for trajectories of drug response and that the response trajectories based on the 2-component DAS28 were identified using a structured methodologic framework (33). In addition, all classes of licensed biologic DMARD treatment regimens were considered and included in longitudinal data models, and the clinical relevance of the identified subgroups was compared using outcomes relevant to patients.

We found that the 7 subgroups demonstrated differing degrees of treatment response, mean patterns of change in disease activity, and clinical characteristics. For example, drug levels were better tracked using the 7 trajectories from the 2-component DAS28 than the 3 trajectories from the 4-component DAS28. Furthermore, antidrug antibody positivity was enriched in 2 of the nonresponder groups using the 2-component disease activity score, indicating that these trajectories are biologically relevant and more informative than those identified using the 4-component score.

Another potentially clinically useful subgroup identified using the 2-component DAS28 was the low disease activity group, in which conventional DAS28 scores were driven by the TJC and VAS. This group was less likely to respond to treatment $(79 \%$ classified as EULAR nonresponders by 6 months) and less likely to adhere to treatment. It is also interesting to note that previous nonadherence, together with an increased resistance to therapy, might be related to a higher proportion of patients receiving previous biologic DMARD treatment (nearly $37 \%$ in the low disease activity group). Use of biologic drugs in this subgroup was unlikely to be clinically efficacious or cost-effective, and biomarkers to identify such patients would be useful.

While the 4-component DAS28 is an important holistic assessment that better captures depression, the 2-component DAS28 appears better able to capture biologic comorbidities, especially respiratory phenotypes. As previously demonstrated (23), antidrug antibody concentrations and treatment adherence remained the most important predictors of drug levels over time. The 2-component trajectories capture these characteristics and confirm previous findings that drug levels, treatment adherence, and antidrug antibody positivity correlate with the speed and sustainability of response.

A number of limitations should be considered. First, while the 2-component DAS28 score was developed to better correlate with synovitis and was validated by demonstrating increased association with radiographic progression in early RA, its weighting remains to be optimized in patients with established RA and those with RA receiving biologic DMARDs, and the implications for treatment decisions have yet to be established. Second, drug levels and antidrug antibody measures were available in only a subset of participants, even if similarly distributed across the 7 trajectory subgroups. Furthermore, adherence to treatments was self-reported. Third, although we assessed baseline comorbidities, adverse events leading to treatment cessation are potential confounders, especially in patients showing high rates of nonadherence, but we did not have sufficient information on those events to include them in the current analysis. A further limitation relates to the selection of participants in the BRAGGSS study, following guidelines from the UK National Institute for Care and Excellence (38). Our findings are therefore generalizable only to populations whose treatments and clinical characteristics are comparable to those outlined in these guidelines.

A final limitation pertains to the exploitation of unsupervised approaches and the exploratory hypothesis-generating nature of these approaches. However, we mitigated this effect by using clinicians' experience and the previous literature (i.e., suggesting 3 classes of responders as measured via the 4-component DAS28, which we replicate in this study), following a robust analytical framework (i.e., testing and retesting multiple models), and performing sensitivity analyses on 2 subsets to verify findings.

In pursuing the characterization of improvement in biologic changes in synovitis, relying solely on the 4-component DAS28 trajectories may result in misclassification of synovial inflammatory responses. The 2-component DAS28 trajectories provide finer granularity and may be a better measurement to reveal underlying biology. While there is currently no method implemented to predict class assignment for new patients, research to identify molecular biomarkers that can discriminate between trajectory classes at baseline will enable this. Thus, we suggest the use of the 2-component DAS28 score for biomarker discovery studies, which should encompass the understanding of adherence to treatment, immunogenicity to drugs, and general health status, including $\mathrm{HAQ}$ scores and comorbidities.

Better defined and physiologically contrasted phenotypes imply a better understanding of the underlying response mechanisms and are the pillar of precision medicine and well-powered biomarker discovery. Previous studies have shown that patients themselves want precision medicine approaches to receive medications that are likely to work more quickly (39). Therefore, more robust prediction models for biologic DMARD responses should be built on the basis of outcome measures reflecting the disease biology, provide evidence of larger variability in responses to biologic treatments, and allow for the identification of essential covariates to build these models. The present study shows how 2-component DAS28 trajectories capture these aspects better than traditional outcome measures and suggests that a more holistic view of patient care, considering all factors likely to influence response, should be applied in order to move toward precision medicine approaches. 


\section{AUTHOR CONTRIBUTIONS}

All authors were involved in drafting the article or revising it critically for important intellectual content, and all authors approved the final version to be published. Dr. Dagliati had full access to all of the data in the study and takes responsibility for the integrity of the data and the accuracy of the data analysis.

Study conception and design. Dagliati, Plant, Geifman, Barton.

Acquisition of data. BRAGGSS Study Group.

Analysis and interpretation of data. Dagliati, Plant, Nair, Jani, Amico, Peek, Morgan, Isaacs, Wilson, Hyrich.

\section{REFERENCES}

1. Giannoula A, Gutierrez-Sacristán A, Bravo Á, Sanz F, Furlong LI. Identifying temporal patterns in patient disease trajectories using dynamic time warping: a population-based study. Sci Rep 2018;8:4216.

2. Jensen AB, Moseley PL, Oprea TI, Ellesøe SG, Eriksson R, Schmock $\mathrm{H}$, et al. Temporal disease trajectories condensed from populationwide registry data covering 6.2 million patients. Nat Commun 2014; 5:4022.

3. Defossez G, Rollet A, Dameron O, Ingrand P. Temporal representation of care trajectories of cancer patients using data from a regional information system: an application in breast cancer. BMC Med Inform Decis Mak 2014;14:24.

4. Joyce DW, Kehagia AA, Tracy DK, Proctor J, Shergill SS. Realising stratified psychiatry using multidimensional signatures and trajectories. J Transl Med 2017;15:15.

5. Edwards RR, Dworkin RH, Turk DC, Angst MS, Dionne R, Freeman $\mathrm{R}$, et al. Patient phenotyping in clinical trials of chronic pain treatments: IMMPACT recommendations. Pain 2016;157:1851-71.

6. Tenenbaum JD, Avillach P, Benham-Hutchins M, Breitenstein MK, Crowgey EL, Hoffman MA, et al. An informatics research agenda to support precision medicine: seven key areas. J Am Med Inform Assoc 2016;23:791-5.

7. Gambhir SS, Ge TJ, Vermesh O, Spitler R. Toward achieving precision health. Sci Transl Med 2018;10:3612.

8. Geifman N, Lennon H, Peek N. Patient stratification using longitudinal data: application of latent class mixed models. Stud Health Technol Inform 2018;247:176-80.

9. Geifman N, Kennedy RE, Schneider LS, Buchan I, Brinton RD. Datadriven identification of endophenotypes of Alzheimer's disease progression: implications for clinical trials and therapeutic interventions. Alzheimer's Res Ther 2018;10:4.

10. Vistisen D, Witte DR, Tabák AG, Herder C, Brunner EJ, Kivimäki M, et al. Patterns of obesity development before the diagnosis of type 2 diabetes: the Whitehall II cohort study. PLoS Med 2014;11:e1001602.

11. Zheng Y, Song M, Manson JE, Biovannucci EL, Hu FB. Group-based trajectory of body shape from ages 5 to 55 years and cardiometabolic disease risk in 2 US cohorts. Am J Epidemiol 2017;186:1246-55.

12. Lin H, Turnbull BW, McCulloch CE, Slate EH. Latent class models for joint analysis of longitudinal biomarker and event process data: application to longitudinal prostate-specific antigen readings and prostate cancer. J Am Stat Assoc 2002;97:53-65.

13. Verger P, Mmadi Mrenda B, Cortaredona S, Tournier M, Verdoux H. Trajectory analysis of anxiolytic dispensing over 10 years among new users aged 50 and older. Acta Psychiatr Scand 2018;137:328-41.

14. Boucquemont J, Loubère L, Metzger M, Combe C, Stengel B, Leffondre K, et al. Identifying subgroups of renal function trajectories. Nephrol Dial Transplant 2017;32 Suppl 2:ii185-93.

15. Signori A, Izquierdo G, Lugaresi A, Hupperts R, Grand'Maison F, Sola $\mathrm{P}$, et al. Long-term disability trajectories in primary progressive MS patients: a latent class growth analysis. Mult Scler 2018;24: 642-52.
16. Barnabe C, Sun Y, Boire G, Hitchon CA, Haroui B, Thorne JC, et al. Heterogeneous disease trajectories explain variable radiographic, function and quality of life outcomes in the Canadian Early Arthritis Cohort (CATCH). PLoS One 2015;10:e0135327.

17. Norton S, Sacker A, Dixey J, Done J, Williams P, Young A, et al. Trajectories of functional limitation in early rheumatoid arthritis and their association with mortality. Rheumatology (Oxford) 2013;52:2016-24.

18. Prevoo ML, van 't Hof MA, Kuper $H H$, van Leeuwen MA, vande Putte LB, van Riel PL. Modified disease activity scores that include twenty-eight-joint counts: development and validation in a prospective longitudinal study of patients with rheumatoid arthritis. Arthritis Rheum 1995;38:44-8.

19. Siemons L, Ten Klooster PM, Vonkeman HE, Glas CA, van de Laar MA. Distinct trajectories of disease activity over the first year in early rheumatoid arthritis patients following a treat-to-target strategy. Arthritis Care Res (Hoboken) 2014;66:625-30.

20. Courvoisier DS, Alpizar-Rodriguez D, Gottenberg JE, Hernandez $\mathrm{MV}$, lannone F, Lie E, et al. Rheumatoid arthritis patients after initiation of a new biologic agent: trajectories of disease activity in a large multinational cohort study. EBioMedicine 2016;11:302-6.

21. RA-MAP Consortium. Novel methodology to discern predictors of remission and patterns of disease activity over time using rheumatoid arthritis clinical trials data. RMD Open 2018;4:e000721.

22. Hensor EM, McKeigue P, Ling SF, Colombo M, Barrett JH, Nam JL, et al. Validity of a two-component imaging-derived disease activity score for improved assessment of synovitis in early rheumatoid arthritis. Rheumatology (Oxford) 2019;58:1400-9.

23. Jani M, Isaacs JD, Morgan AW, Wilson AG, Plant D, Hyrich KL, et al. High frequency of antidrug antibodies and association of random drug levels with efficacy in certolizumab pegol-treated patients with rheumatoid arthritis: results from the BRAGGSS cohort. Ann Rheum Dis 2017;76:208-13.

24. Jani $M$, Chinoy $H$, Warren $R B$, et al. Clinical utility of random antitumor necrosis factor drug-level testing and measurement of antidrug antibodies on the long-term treatment response in rheumatoid arthritis. Arthritis Rheumatol 2015;67:2011-9.

25. Cordingley L, Prajapati R, Plant D, Maskell D, Morgan C, Ali FR, et al. Impact of psychological factors on subjective disease activity assessments in patients with severe rheumatoid arthritis. Arthritis Care Res (Hoboken) 2014;66:861-8.

26. DAS 28. URL: https://www.das-score.nl/das28/en/.

27. Fries JF, Spitz PW, Kraines RG, Holman HR. Measurement of patient outcome in arthritis. Arthritis Rheum 1980;23:137-45.

28. Verheij RA, Curcin V, Delaney BC, McGilchrist MM. Possible sources of bias in primary care electronic health record data use and reuse. J Med Internet Res 2018;20:e185.

29. Weber GM, Adams WG, Bernstam EV, Bickel JP, Fox KP, Marsolo K, et al. Biases introduced by filtering electronic health records for patients with "complete data." J Am Med Inform Assoc 2017;24:1134-41.

30. Dagliati A, Marini S, Sacchi L, Cogni G, Teliti M, Tibollo V, et al. Machine learning methods to predict diabetes complications. J Diabetes Sci Technol 2018;12:295-302.

31. Celton M, Malpertuy A, Lelandais G, de Brevern AG. Comparative analysis of missing value imputation methods to improve clustering and interpretation of microarray experiments. BMC Genomics 2010;11:15.

32. Proust-Lima C, Philipps V, Liquet B. Estimation of latent class linear mixed models: the new package Icmm. J Statistical Softw 2017;78:1-56

33. Lennon H, Kelly S, Sperrin M, Buchan I, Cross AJ, Leizmann M, et al. Framework to construct and interpret latent class trajectory modelling. BMJ Open 2018;8:e02083. 
34. Lenth RV. Least-squares means: the R package Ismeans. J Stat Softw 2016;69:1-33.

35. Ling S, Bluett J, Barton A. Prediction of response to methotrexate in rheumatoid arthritis [review]. Expert Rev Clin Immunol 2018;14: 419-29.

36. Ottaviani S, Gardette A, Tubach F, Roy C, Palazzo E, Gill G, et al. Body mass index and response to infliximab in rheumatoid arthritis. Clin Exp Rheumatol 2015;33:478-83.

37. Van Gestel AM, Prevoo ML, van 't Hof MA, van Rijswijk $M H$, van de Putte LB, van Riel PL. Development and validation of the European League Against Rheumatism response criteria for rheumatoid arthritis: comparison with the preliminary American College of Rheumatology and the World Health Organization/International League Against Rheumatism criteria. Arthritis Rheum 1996;39:34-40.

38. National Institute for Health and Care Excellence. Drug treatment for rheumatoid arthritis. URL: https://pathways.nice.org.uk/pathways/ rheumatoid-arthritis\#path=view\%3A/pathways/rheumatoidarthritis/drug-treatment-for-rheumatoid-arthritis.xml\&content=viewindex.

39. Kumar K, Peters S, Barton A, on behalf of MATURA. Rheumatoid arthritis patient perceptions on the value of predictive testing for treatments: a qualitative study. BMC Musculoskelet Disord 2016;17:460. 